\title{
Comparison of results from turbulence models for the Nomad flaperon-configured aerofoil
}

\author{
$\begin{array}{ll}\text { D. Norrison } & \text { E. Ly } \\ & \end{array}$
}

(Received 1 August 2006; revised 12 December 2007)

\begin{abstract}
Incidents involving low speed flutter of the wing-flaperon configured Government Aircraft Factories Nomad N22 and N24 have been reported. Wind tunnel experiments are unable to reproduce the effect. To better understand what could have caused the reported flutter, a computational fluid dynamics based investigation was undertaken, employing a complex commercial simulation software, with the aim to study the local flowfield structures around this configuration, focusing specifically on turbulence effects. Inviscid and viscous flows incorporating the Spalart-Allmaras and RNG $k$ - $\epsilon$ turbulence models were computed. Comparisons with experimental data showed excellent agreement among the results, with the predicted section lift coefficients being within $10 \%$ of the measured values.
\end{abstract}

See http://anziamj.austms.org.au/ojs/index.php/ANZIAMJ/article/view/65 for this article, (c) Austral. Mathematical Soc. 2007. Published December 17, 2007. ISSN 1446-8735 


\section{Contents}

1 Introduction

C592

2 Numerical flow simulations $\quad$ C593

2.1 Turbulence models . . . . . . . . . . . . . . . C597

3 Computed results and discussions

C599

4 Conclusion

C601

References

C602

\section{Introduction}

Flutter has been suspected to affect the flaperon-configured aerofoil of the Government Aircraft Factories' (GAF) Nomad aircraft. The Nomad family consists of two aircraft notably the N22 and its variant the N24, which is a stretched version of the N22 type model. The twin turboprop, high wing Nomad aircraft was designed primarily as a short take off and landing (STOL) utility aircraft for short and medium range transportation of up to thirteen passengers (with or without cargo). Its remarkable STOL capability was attributed to its engines and full span flaperon configuration [11, 15].

Flaperons, also known as drooped ailerons, are wing control surfaces where the ailerons (which normally move in opposite directions to one another causing the aircraft to roll longitudinally) are both lowered equally to assist the flaps in generating sufficient lift for an aircraft to fly at low speeds, specifically during the take off and landing phases [13]. In these flight conditions, it is suspected that flutter affects the Nomad's flaperons and its overall flight performance. Flutter is an aerodynamically induced vibration of the wing, tail or other part of the aircraft, which can result in a 
complete structural failure. However, it is uncommon for flutter to occur at low flight speeds. Numerous attempts to reproduce the flutter effects under a controlled environment in wind tunnel tests have been unsuccessful. Yet, flutter has been observed in real flights $[4,5,7]$. In order to avoid this flutter, performance limitations have been imposed upon the Nomad preventing it from operating as originally intended. These include flight speed restrictions and to refrain from using the $38^{\circ}$ flap setting [6].

To better understand what could have caused the reported flutter, a computational fluid dynamics (CFD) based investigation, utilising a commercial simulation software FLUENT, was undertaken to study the local flowfield structures around this configuration; focusing specifically on turbulence effects and the flow through the spaces between the main aerofoil and the two flaps. The problem was simplified by limiting the investigation to a two dimensional static aerofoil section and selecting cases with experimental data. The spoiler present on the outboard wing section was not modelled, so only inboard data was used [8]. This investigation serves as a starting point for more detailed analyses. The computed inviscid and viscid solutions compare very well with the experimental data, with the difference between the predicted and measured section lift coefficients being less than $10 \%$.

\section{Numerical flow simulations}

Two cases were studied: Case $0 / 0$ with both flaps at $0^{\circ}$, angle of attack, $\alpha$, of $16^{\circ}$, and an airspeed of $63 \mathrm{~m} / \mathrm{s}$ or 123 knots; Case 30/60 with front and rear flaps at $30^{\circ}$ and $60^{\circ}$, respectively, $12^{\circ}$ angle of attack, and an airspeed of $44 \mathrm{~m} / \mathrm{s}$ or 85 knots. Case $30 / 60$ was of particular interest as it resembled the landing configuration and conditions for which flutter was reported.

The three element aerofoil, shown in Figure 1, is based on a NACA 23018 profile [1] with modifications around the leading edge (LE) and trailing edge (TE) where deviating coordinates were measured from a $50 \%$ scale engineering 



Figure 1: Nomad aerofoil for Case 0/0 (left) and Case 30/60 (right).

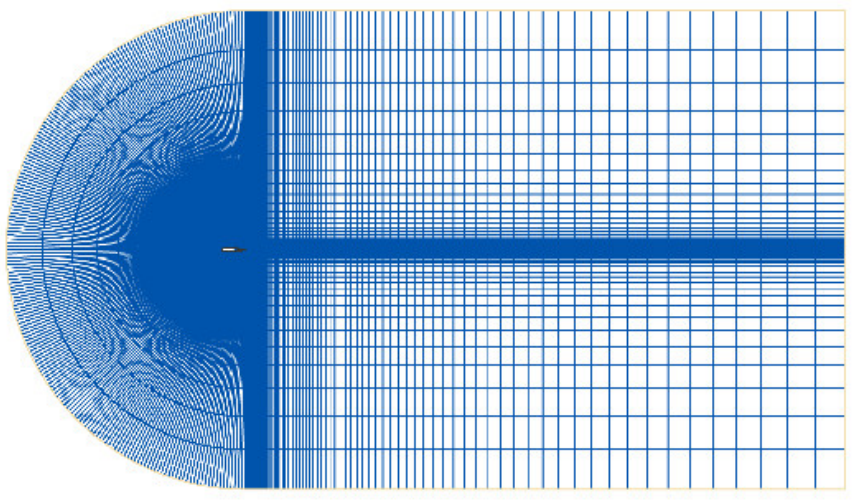

Figure 2: C-grid used in the FLUENT simulations. 


\begin{tabular}{ccc} 
TABLE 1: Inviscid flow section lift coefficient \\
\hline Mesh & $c_{l}$ (Case 0/0) & $c_{l}$ (Case 30/60) \\
\hline Coarse & 1.694 & 3.109 \\
Medium & 1.737 & 3.101 \\
Fine & 1.758 & NA \\
\hline
\end{tabular}

drawing of the wing [9]. The main element, front and rear flaps consisted of 195, 69 and 80 data points, respectively, which were nondimensionalised using the aerofoil chord length, $\ell$. The C-grid in Figure 2 was used, with a $10 \ell$ radius arc centred at the $\mathrm{TE}$, and a $25 \ell$ (streamwise) by $20 \ell$ (vertical) boundary behind the TE.

Three meshes with increasing cell density were used to confirm that the predicted results were grid independent for the inviscid flow solution. The coarse, medium and fine meshes contained approximately 15,000, 23,000 and 35,000 cells, respectively. This roughly equated to a $50 \%$ increase in cell numbers for each successive mesh, with the number of nodes being scaled appropriately. For the coarse mesh, the main aerofoil element, front and rear flaps consisted of 150, 70 and 60 nodes, respectively, and 10 streamwise nodes for the gaps between the aerofoil elements. The region behind the TE consisted of 84 nodes in the vertical direction and 122 streamwise nodes. Meshing software, GAMBIT (version 2.2.3), produced the structured meshes using quadrilateral elements. With grid independent results for inviscid flow, see Table 1, the coarse mesh was deemed adequate for the simulations. The lift coefficient for the Case 30/60 fine mesh was absent as it did not converge after one million iterations. Since the other meshes converged in under 40,000 iterations, it seemed unlikely that a converged solution would be obtained with more iterations. Further investigation is warranted to identify the cause, which is suspected to be mesh related.

All simulations were performed on a computer with a Pentium $4,2.4 \mathrm{GHz}$ processor, $512 \mathrm{Mb}$ physical memory, $720 \mathrm{Mb}$ virtual memory, and Microsoft 
Windows XP Professional SP2 installed. The two dimensional double precision version of FLUENT (version 6.2.16) was used, executing the steady implicit coupled solver, together with the energy equation. The governing equations employed by the solver are the Euler and Reynolds-averaged Navier-Stokes equations for inviscid and viscous flows, respectively, available in aerodynamics texts $[2,16]$. Pressure farfield boundary conditions were applied at sea level conditions (gauge pressure of $101,325 \mathrm{~Pa}$ and temperature of $288.16 \mathrm{~K}$ ), and air density was treated as an ideal gas. When a turbulence model was used, default values were adopted, with the exception that the turbulence specification method was set to a turbulent viscosity ratio of 10 , and the three coefficient Sutherland law for air viscosity was used. For the solver, the modified turbulent viscosity was set to a second order, upwind, discretisation scheme. The Courant number was initially set to 1.25 for the first 200 iterations to get the solution started and, to accelerate convergence, it was raised to 5 with 4,800 iterations performed. If the solution diverged, the simulation was restarted with a reduced Courant number, as low as 0.5 for the first 200 iterations and 2 for the remaining iterations. If necessary, additional blocks of 5,000 iterations were performed until converged. Convergence was achieved by setting the residual limits to a low value $\left(10^{-6}\right.$ or less), and inspecting the residual and $c_{l}$ versus iterations history plots. No further iterations were performed once the $c_{l}$ and residuals had reached a steady state as illustrated in Figure 3.

The $c_{l}$ values reported here (see Figure 4) for each case were manually calculated based solely on the pressure coefficient $\left(c_{p}\right)$ distributions, neglecting the effect of skin friction, which was assumed to be very small. This allowed a direct comparison with the $c_{l}$ values stated in the Nomad documentation (which were computed with in-house software using wind tunnel pressure data [10]). The $c_{l}$ values presented here were for the main aerofoil element only for which experimentally measured pressure data was provided. 



Figure 3: Convergence history for Case 30/60 (inviscid flow and coarse mesh).

\subsection{Turbulence models}

The flowfield around the Nomad aerofoil was solved for inviscid flow as well as for viscous flow, so that the turbulence effects due to the flap elements could be studied along with the region downstream of the TE. Five different turbulence models can be selected in FLUENT, notably Spalart-Allmaras (sA), $k-\epsilon, k-\omega$, Reynolds stress and large eddy simulation (which is available only in the three dimensional version of FLUENT). Their purpose is to model the effects of turbulence when the grid used to model a problem is too coarse to compute the large and small scale structure, associated with turbulent flow, directly from the Navier-Stokes equations, a method better known as direct numerical simulation (DNS). In all but a few simple cases, it is impractical to use DNS as an exceptionally fine grid is required which consequently requires enormous amounts of computer power and time to solve. Therefore, for simplicity and as a first step in investigating the turbulence effects for the current research work, the most simple turbulence models ( $\mathrm{S}-\mathrm{A}$ and $k-\epsilon$ ) were employed for the viscous flow simulations.

The S-A model formulated in 1992 [14] employs only one transport equa- 

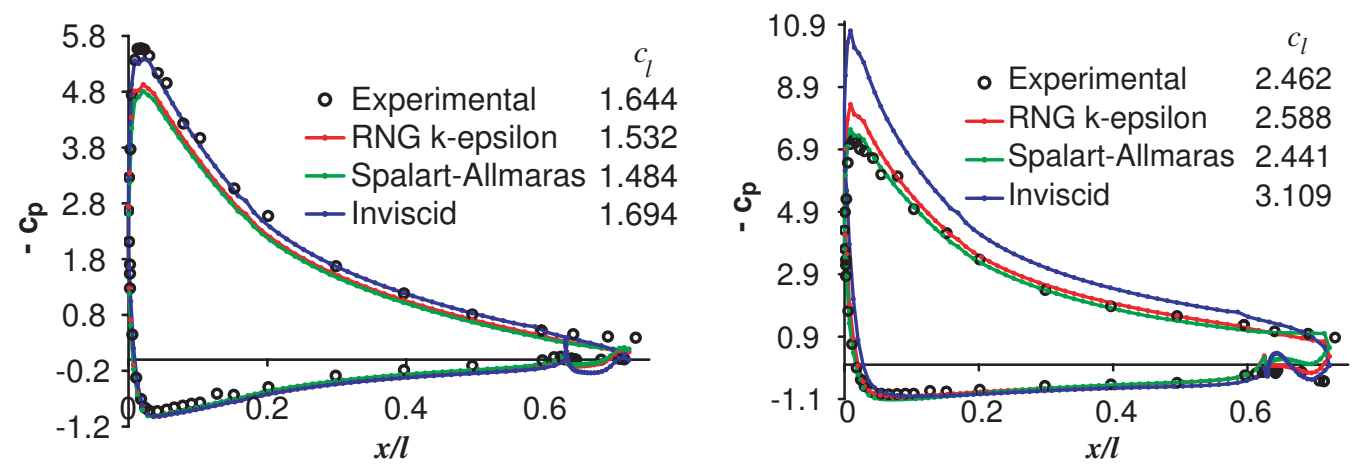

Figure 4: Pressure coefficient distribution with $c_{l}$ values for Nomad main aerofoil element for Case 0/0 (left): $\alpha=16^{\circ}, M_{\infty}=0.186, \operatorname{Re}_{\infty}=4.33\left(10^{6}\right)$; and Case 30/60 (right): $\alpha=12^{\circ}, M_{\infty}=0.129, \operatorname{Re}_{\infty}=2.99\left(10^{6}\right)$.

tion to represent the turbulent viscosity, $\mu_{t}$. It was designed to suit aerofoil and wing applications, making it desirable for analysis. However, a turbulence model where velocity and length scale transport effects are modelled individually may sometimes be preferred.

The $k$ - $\epsilon$ model devised in 1972 [12] is one such model, using two transport equations to represent $\mu_{t}$. It is often used in engineering problems since it performs well for a broad range of turbulent flows. Two variants with better performance are offered in FLUENT, notably the renormalisation group (RNG) and realisable $k-\epsilon$ models. The RNG $k-\epsilon$ model was adopted due to its improved performance in flow areas where vortices and rotation are present. Nonequilibrium wall functions were deemed appropriate given the flow separation expected for the cases considered in this investigation. 

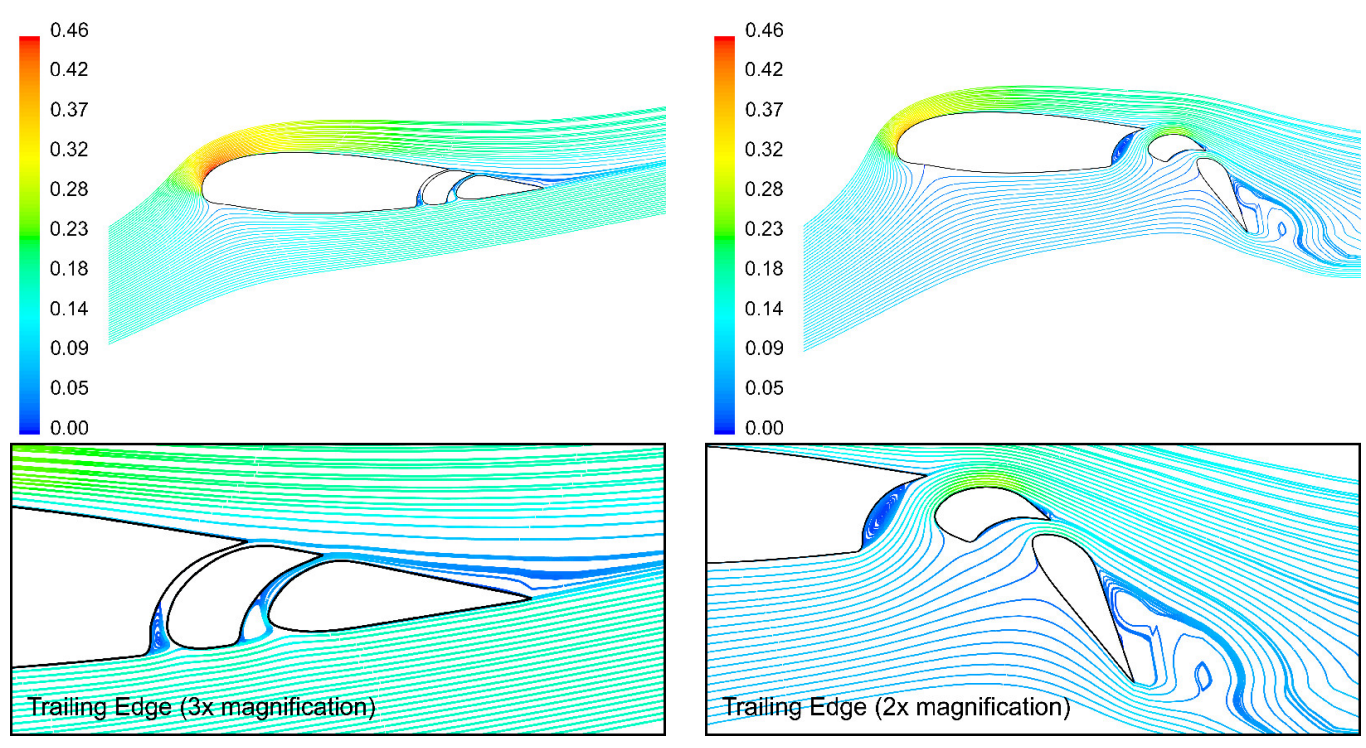

FiguRE 5: Local Mach number coloured streamline plots for the Nomad aerofoil for Case $0 / 0$ (left): $\alpha=16^{\circ}, M_{\infty}=0.186, \operatorname{Re}_{\infty}=4.33\left(10^{6}\right)$; and Case 30/60 (right): $\alpha=12^{\circ}, M_{\infty}=0.129, \operatorname{Re}_{\infty}=2.99\left(10^{6}\right)$.

\section{Computed results and discussions}

Figure 4 displays the predicted pressure coefficient distributions along the main aerofoil element for both cases, where $x$ is the distance along the chord, and the $\mathrm{TE}$ and $\mathrm{LE}$ are located at $x / \ell=0$ and $x / \ell=1$ respectively. It was anticipated that the inviscid solution would produce a much higher $c_{l}$ than that from experiment, but for Case $0 / 0$ it agrees more closely than for the viscous solutions. The relative difference between the experimentally measured $c_{l}$ and the inviscid $c_{l}$ was about $3.0 \%$ as opposed to $6.8 \%$ and $9.7 \%$ for the $c_{l}$ values obtained when the RNG $k-\epsilon$ and S-A turbulence models were used. The overall trend of the predicted and experimental pressure distributions is good, except for the region near the TE. 
Case 30/60 produced viscous results that compared favourably with the experimental data, while the inviscid solution significantly over estimated the section lift coefficient. In regards to the s-A model, the flowfield around the LE was well captured, except for a very small region on the concave side of the TE where a vortex was generated. The RNG $k-\epsilon$ turbulence model produced a pressure distribution trend that was more akin to experiment, which was hoped for due to its better performance in simulating flowfields with separation and vortices. Yet, at the upper side of the LE it overestimated the pressure when compared to experiment and the S-A model. Overall, the RNG $k-\epsilon$ turbulence model with nonequilibrium wall functions generated results that best represented the experimental data. When the experimental $c_{l}$ was compared to the predicted inviscid $c_{l}$ the relative difference was about $26.3 \%$, as opposed to $5.1 \%$ and $0.9 \%$ for the RNG $k-\epsilon$ and S-A turbulence models respectively.

Since the RNG $k-\epsilon$ turbulence model produced better results than the S-A model in the TE region where vortices were present, it was used to model streamlines around the aerofoil. The turbulence and flowfield structure are clearly revealed for both cases by the streamline plots in Figure 5, which are coloured by the local Mach numbers. Inspection of this figure shows that for Case $0 / 0$, small vortices were generated in the region behind the main aerofoil element and the front flap. The flow remained attached along the upper and lower aerofoil surfaces except for the gaps between the flaps. For Case 30/60, all three aerofoil elements had vortices present at the TE, with a large vortex behind the main aerofoil element and the rear flap, and a very small vortex on the upper side of the front flap. This observation is similar to that observed by Chakrabartty, Mathur and Dhanalakshmi [3] for a similarly configured wing.

The meshes used here were not deemed ideal, due to difficulties in meshing the spaces between the flaps. At the junction where the aerofoil would normally be if flaps were not present, the vertical grid lines did not smoothly adjust to the corresponding lines within the flap gap region. Furthermore, for 
Case 30/60, the mesh should have left the TE of the rear flap at almost the same angle as the flap was directed, rather than being horizontal as shown in Figure 1. An attempt to rectify these problems, and minimise skewed cells, by using an unstructured grid with triangular elements was unsuccessful. This was because GAMBIT's sizing function did not work as anticipated for the problem. When applied to the aerofoil, the only parameter which influenced the mesh was the start size. To have a sufficiently fine mesh near the aerofoil boundary the start size was made small and resulted in an unacceptably fine mesh being produced in a large region (about $6 \ell$ to $7 \ell$ radius) around the aerofoil, since the growth rate seemed to be ignored.

\section{Conclusion}

Flowfields around the GAF Nomad aircraft aerofoil were simulated using FLUENT (an advanced commercial CFD package). The best results were obtained with the RNG $k-\epsilon$ turbulence model, with excellent agreement between the predicted section lift coefficient and the experimentally measured one, with a relative difference of less than $7 \%$. In future work, the problems associated with the grids will be rectified by using our own grid generation computer code, and the work will be extended to three dimensions. Furthermore, other turbulence models such as the LES model will be employed for simulations to advance our understanding of the turbulence effects for the flaperon-configured aerofoil.

Acknowledgements: The authors thank Mr Rob Danaher, Hawker de Havilland, Melbourne, Australia, for his support and providing the GAF Nomad technical documentation used in this report. 


\section{References}

[1] Abbott, I. H., and Von Doenhoff, A. E., Theory of Wing Sections: Including a Summary of Airfoil Data, Dover Publications, Inc., New York, USA, 1959. C593

[2] Anderson, J. D., Fundamentals of Aerodynamics, Third Edition, McGraw-Hill, Singapore, 2001. C596

[3] Chakrabarty, S. K., Mathur, J. S., and Dhanalakshmi, K., Applications of Advanced CFD Codes for Aircraft Design and Development at NAL, Journal of Aerospace Sciences \& Technologies, Technical Note, Volume 55, Number 1, Feb 2003, pp. 74-88. C600

[4] Civil Aviation Safety Authority (CASA), Aileron Structure: Schedule of Airworthiness Directives, AD/GAF-N22-64, Australia, 1992, website: http://www.casa.gov.au/airworth/airwd/ADfiles/ under/gaf-n22/GAF-N22-064.pdf. C593

[5] Civil Aviation Safety Authority (CASA), Ailerons: Schedule of Airworthiness Directives, AD/GAF-N22-69 Amdt 4, Australia, 2003, website: http://www.casa.gov.au/airworth/airwd/ADfiles/ under/gaf-n22/GAF-N22-069.pdf. C593

[6] Civil Aviation Authority (CAA), Airworthiness Directives Schedule: GAF Nomad N22/24 Series, Australia, 2004, pp. 13, website: http://www.caa.govt.nz/fulltext/nzcars/vol_2/Section_A/A1_ Aeroplanes/n22.pdf. C593

[7] Farrell, P. A., and Dunn, S. A., Flight Flutter Test of a Nomad N24A Aircraft Fitted with One Modified Aileron, Aeronautical and Maritime Research Laboratory (AMRL), Defence Science and Technology Organisation (DSTO), Melbourne, Australia, 1995, website: http:// Www.dsto . defence.gov. au/publications/2630/DSTO-TN-0012.pdf. C593 
[8] Government Aircraft Factories (GAF), Nomad: Project N2 Certification Report 1020 Load Summary, Australia, 1970. C593

[9] Government Aircraft Factories (GAF), Drawing Number: N-20-5, Australia, circa 1970. C595

[10] Government Aircraft Factories (GAF), Nomad: Certification Report N22-9101 Volume 2 Aerodynamic Load Analysis - Two dimensional Wind Tunnel Tests, Australia, 1977. C596

[11] Government Aircraft Factories (GAF), Nomad: the Australian N22B and N24A Turboprop, Australian Government Publishing Service, Australia, 1981. C592

[12] Launder, B. E., and Spalding, D. B., Lectures in Mathematical Models of Turbulence, Academic Press, London, England, 1972. C598

[13] McCormick, B. W., Aerodynamics, Aeronautics and Flight Mechanics, Second Edition, John Wiley \& Sons, Inc., USA, 1995. C592

[14] Spalart, P., and Allmaras, S., A One-Equation Turbulence Model for Aerodynamic Flows, American Institute of Aeronautics and Astronautics (AIAA), AIAA Paper 92-0439, 1992. C597

[15] Taylor, M. J. H., Jane's Encyclopedia of Aviation, Portland House, USA, 1989. C592

[16] Wilcox, D. C., Turbulence Modeling for CFD, Second Edition, DCW Industries, USA, 2000. C596 


\section{Author addresses}

1. D. Norrison, School of Mathematical and Geospatial Sciences, RMIT University, Melbourne, Victoria 3001, Australia. mailto:daniel.norrison@rmit.edu.au

2. E. Ly, School of Mathematical and Geospatial Sciences, RMIT University, Melbourne, Victoria 3001, Australia. 